\title{
Relationship between prematurity, high blood pressure and kidney injury
}

\begin{abstract}
Introduction: Prematurity is a public health problem worldwide. Reducing the morbidity and mortality of prematuritythroughimprovedmanagementtechniquesrevealtheemergenceof cardiovascular risk diseases, including kidney disease and high blood pressure, which can compromise health in adulthood. The prevalence of these diseases varies from country to country. The main objective of this study was to determine the factors associated with renal injury and high blood pressure in children born preterm.
\end{abstract}

Methodology: We conducted a historical cohort study including children aged 6 to 11 years, matched for gender and sex in a 1:2 ratio. Data were collected from the records of premature and full-term infants hospitalized from January 1, 2008 to December 31, 2013. Patients were reviewed during outpatient consultation, where height, blood pressure were measured and a urine dipstick was performed. Patients with high blood pressure or positive protein uria were seen again two weeks later for follow-up. The Fisher test and the Chisquare test were used to compare proportions. The significance threshold was defined for $p<0.05$. Relative risk (RR) was used to establish the risk relationship between the different variables.

Results: We enrolled 125 children born preterm and 250 born at full-term. The mean age was $8.2+/-1.6$ years with discrete female predominance. The cumulative incidence of pathologies varied according to type: $17.60 \%$ in preterm versus $2 \%$ in full-term $(p<0,001)$ for high blood pressure and $69.60 \%$ in preterm versus $33.20 \%$ in full-term children $(\mathrm{p}<0,001)$ for proteinuria. Factors associated with renal in jury were the use of amino glycosides, aminophylline and neonatal infection. We found a correlation between the presence of proteinuria and high blood pressure $(\mathrm{r}=1.14, \mathrm{p}=0.000002)$.

Conclusion: Renal damage and high blood pressure were more common in children born preterm than in full-term. Associated factors were neonatal infection, use of amino glycosides and aminophylline.

Keywords: high blood pressure, prematurity, kidney disease, Yaoundé
Volume 10 Issue 4 - 2020

\author{
Evelyn Mah, ${ }^{1,2}$ Suzanne Ngo Um Sap, 1,3 Dany \\ Hermann Ngwanou,' Daniel Kago Tague, ${ }^{1,2}$ \\ Linda Maguip,' David Chelo, 1,3 Temgoua \\ Ngou Mazou,' Gides Zantia,' Andréas \\ Chiabi ${ }^{1,2}$ \\ 'Faculty of Medicine and Biomedical Sciences, the University of \\ Yaoundé I, Cameroon \\ ${ }^{2}$ Yaoundé Gynaeco-Obstetrics and Pediatric Hospital, \\ Cameroon \\ ${ }^{3}$ Mother and Child Center, Chantal Biya Foundation, Cameroon
}

Correspondence: Kago Tague Daniel Armand, Faculty of Medicine and Biomedical Sciences, the University of Yaoundé I, Yaounde, Cameroon, Tel +(237) 6771794 64, Email kagog@yahoo.fr

Received: July 06, 2020 | Published: July 20, 2020

\section{Introduction}

Prematurity, a major public health problem is the second leading cause of neonatal mortality in developing countries. In combination with intrauterine growth retardation, prematurity is now an indicator of the general health status of newborns, and a determinant key of child survival, health and development. ${ }^{1}$ In some Western countries, the frequency of prematurity varies from 5 to $9 \%^{2}$ while in Africa, it is estimated to be above $15 \% .{ }^{3,4}$ In Cameroon, the premature birth rate was $13 \%$ in $2010 .{ }^{5}$ At the Yaoundé Gynaeco- Obstetric and Pediatric Hospital in 2011, 26.5\% of newborns admitted to the neonatology unit were premature. ${ }^{6}$ The newborn, regardless of birth weight and/ or gestational age, is a fragile being; this fragility increases when birth occurs before term, with the potentially serious consequences in the short, medium and long term. Advances in perinatal medicine and neonatology have improved the survival of premature infants. Despite the benefits of these advances in terms of reducing mortality, premature infants are at risk of developing chronic non-communicable diseases in the long term, including kidney disease and high blood pressure, increasing subsequent cardiovascular risk. ${ }^{7}$ The frequency of these diseases is increasing in Europe and Asia, but not systematically assessed in our context. This could explain the scarcity of data on the subject in sub-Saharan Africa in general and Cameroon in particular.
The main objective was to determine the factors associated with the occurrence of high blood pressure and renal disease in premature infants at the Yaoundé Gyneco-Obstetrics and Pediatric Hospital. This study will provide us with data on the extent of the problem in our context and the associated risk factors; in order to raise attention of health personnel to look for those diseases during the follow up of premature infants and the early management of detected cases, thus contributing to the reduction of preventable morbidity and mortality in adulthood.

\section{Materials and method}

We conducted a historical cohort study over a 6-year period from January 2008 to December 2013, including patients aged 6 -11yearsby June 2019.Were included all newborns regardless of gender and Gestational age, admitted to the neonatal unit during the study period and discharged alive. The exposure factor considered was prematurity. Thus two groups were constituted: those born prematurely and those born at full-term and admitted immediately after their respective premature and matched for age and sex. We excluded all small for gestational age, congenital malformations and incomplete records. The minimum sample size was 122 patients, using the formula for cohort study proposed by Charan and Biswas in 2013. ${ }^{8}$ 


$$
\text { Samplesize }=\frac{\left[\begin{array}{c}
Z_{\alpha} \sqrt{\left(1+\frac{1}{m}\right)} p^{*}\left(1-p^{*}\right)+Z_{\beta} \sqrt{p^{1}} \\
\left(1-p^{1}\right) / m+p^{2}\left(1-p^{2}\right)
\end{array}\right]^{2}}{\left(p_{1}-p_{2}\right)^{2}}
$$

As part of the procedure, after obtaining the research authorizations requested from the competent authorities, we went through the neonatal admission registers where we listed the names of the patients to be included in the study. Then we searched files in the "archives" unit and incomplete files or files that did not have a telephone number at this stage were excluded.

Then parents were contacted by phone to make an appointment for a consultation. After obtaining the informed consent of the parents, the consultation was done, during which parameters such as the height, weight and the right arm Blood pressure was taken after a 5-minute rest (three times). Concomitantly, a urine deep-stick (colorimetry method) test was performed. Children with neither High blood pressure nor proteinuria continued the usual follow-up according to a schedule proposed to them. Patients with high blood pressure or positive proteinuria were reviewed 15 days later for re-assessment. The pathological cases were referred to the appropriate specialist for optimal follow-up.

The data were analysed by the Epi-Info ${ }^{\circledR}$ software version 3.5.4. For the comparison of the means in the different groups, the Annova test was used when the distribution was symmetric and the Kruskal Wallis test when it was not. The relative risk (RR) with its $95 \%$ confidence interval was used to establish the strength of the association between the different variables. The Pearson correlation test was used to assess the positive or negative correlation between two quantitative variables. The $P$ value was calculated by the Fisher test when the number of patients was less than 5 and the Chi-square test was used in the other cases to compare the proportions. The significance threshold was defined for $P<0.05$. We defined pre-high blood pressure as a blood pressure between $90^{\text {th }}-95^{\text {th }}$ percentile of blood pressure according to the sex, height and age after three measurements. High blood pressure was defined as blood pressure greater than $95^{\text {th }}$ percentile.

\section{Ethical considerations}

The ethical clearances of the Institutional Ethics and Research Committee of the Faculty of Medicine of the University of Yaounde 1 and the Gynaeco-Obstetrics and Paediatric Hospital of Yaoundé were obtained before the onset of the study. The data collected were kept strictly confidential and used only for the purposes of the study.

\section{Results}

We enrolled 125 children born preterm and 250 born at term matched for age and gender.

\section{Socio-demographic data of children}

The average age of patients was $8.2 \pm 1.6$ years with extremes of 6-11 years. The female sex predominated with a sex ratio of 0.98 . Exposed and non-exposed children were comparable for age $(\mathrm{p}=0.98)$ and $\operatorname{sex}(\mathrm{p}=0.6)$.

\section{Socio-demographic data of mothers}

The average age of the mothers was $28.38+/-6.32$ with extremes ranging from 17 to 43 years. The majority were single, primiparous and employed.

\section{Birth history}

The mean gestational age was $33.26+/-2.18$ with extremes ranging from 28 and $36 \mathrm{AS}$, and an average birth weight of $1940 \mathrm{~g}$ +/- 456 (Table 1).

Table I Distribution of the population by pernatal history

\begin{tabular}{lll}
\hline Variables & Preterm infants n= I 25 (\%) & Full-term $\mathbf{n = 2 5 0}(\%)$ \\
\hline Mode of delivery & & $143(57.20)$ \\
Lowtrack & $47(37.60)$ & $107(42.80)$ \\
Caesarean section & & $167(66.80)$ \\
Place of delivery & $91(72.80)$ & $83(33.20)$ \\
YGOPH & $34(27.20)$ & - \\
Otherhospitals & & - \\
Gestationalage in weeks & $\mid(0.80)$ & $250(100)$ \\
$<28$ & $50(40.0)$ & \\
$28-32$ & $74(59.20)$ & $0(0)$ \\
$>32$ & & $20(8.0)$ \\
APGAR score at the 5th minute & $0(0)$ & $230(92.0)$ \\
$<3$ & $9(7.20)$ & \\
$3-6$ & $116(92.80)$ & \\
$>6$ & & \\
\hline
\end{tabular}




\begin{tabular}{lll} 
Table Continued... & Preterm infants $\mathbf{n = 1 2 5}(\%)$ & Full-term $\mathbf{n = 2 5 0}(\%)$ \\
\hline Variables & & \\
\hline Birth weight in grams & $3(2.40)$ & - \\
$<1000$ & $82(65.60)$ & - \\
$1000-2000$ & $40(32.0)$ & $250(100)$ \\
$>2000$ & &
\end{tabular}

\section{Diagnosis at admission}

The main pathologies presented by the studied population were infectious risks (54 preterm versus 99 full-term), neonatal bacterial infections ( 50 preterm versus 49 full-term) and neonatal asphyxia ( 8 preterm versus 28 full-term). The median length of hospitalization was 9.5 (4-15) days with extremes ranging from one to 45 days.

\section{Treatment received during hospitalization}

Treatment received during hospitalization was dominated by antibiotics, including amino sides (99 preterm versus 129 full-term), with a median duration of antibiotics of 8 days (3-10) and extremes ranging from 3 to 19 days. The average duration of amino side in our study was 7 days. We also found significant use of cimetidine (48 preterm versus 13 full-term) and aminophylline (63 preterm only).

\section{Summary of measured parameters}

The average body mass index was $17.99 \mathrm{Kg} / \mathrm{m}^{2}+/-3.24$ with extremes ranging from 10.71 to $29.59 \mathrm{Kg} / \mathrm{m}^{2}$. Systolic blood pressure was high in nearly 72 patients with an average of $113.60 \mathrm{~mm} \mathrm{Hg+/-}$ 11.58. Extremes: $88-141 \mathrm{~mm} \mathrm{Hg}$. Diastolic blood pressure was high in approximately 40 patients, with an average of $67.24 \mathrm{mmHg}+/-$ 10.63. Extremes: $40-90 \mathrm{~mm} \mathrm{Hg}$. About 87 patients had proteinuria that remained positive after two tests at two weeks interval. Overweight was found in nearly 30 patients (23\%) (Table 2 ).

Table 2 Cardiovascular profile of the study population

\begin{tabular}{|c|c|c|}
\hline Variable & Preterm infants $n=\mid 25(\%)$ & Full term $n=250(\%)$ \\
\hline \multicolumn{3}{|c|}{ Proteinuria $(n=87, n=83)$} \\
\hline Traces & $6(4.80)$ & $10(4.0)$ \\
\hline+ & $66(52.8)$ & $63(25.2)$ \\
\hline++ & $15(12.0)$ & $10(4.0)$ \\
\hline \multicolumn{3}{|l|}{ Systolic BP } \\
\hline$<90^{\text {th }}$ percentile & $53(42.4)$ & $211(84.4)$ \\
\hline $90-95^{\text {th }}$ percentile & $50(40.0)$ & $34(13.6)$ \\
\hline$>95^{\text {th }}$ percentile & $22(17.6)$ & $5(2.0)$ \\
\hline \multicolumn{3}{|l|}{ Diastolic PA } \\
\hline$<90^{\text {th }}$ percentile & $86(68.8)$ & $227(90.8)$ \\
\hline $90-95^{\text {th }}$ percentile & $30(24.0)$ & $20(8.0)$ \\
\hline$>95^{\text {th }}$ percentile & $9(7.2)$ & $3(1.2)$ \\
\hline \multicolumn{3}{|c|}{ BMI/age (Z-Score) } \\
\hline$>3$ & $3(2.4)$ & $0(0.0)$ \\
\hline$>2$ & $37(29.6)$ & $20(8.0)$ \\
\hline Between -2 and 2 & $81(64.8)$ & $225(90.0)$ \\
\hline$<-2$ & $3(3.4)$ & $5(2.0)$ \\
\hline$<-3$ & I $(0.8)$ & $0(0.0)$ \\
\hline
\end{tabular}

\section{Profile of pathologies found}

\section{Cumulative incidence of the different pathologies in the two study populations}

Renal injury, marked here by positive proteinuria after two controls, was significantly higher in preterm $(p<0.001)$, likewise
High blood pressure $(\mathrm{p}<0.001)$. The main co morbidity found was overweight, which was also significantly higher in children born preterm $(p=0.00002)$ (Table 3$)$. Overall, these pathologies were found mainly in children born preterm, who had a birth weight between 1000 and 2000 grams (57 cases for renal damage and 14 cases for high blood pressure) and were born after the $32^{\text {nd }}$ week of amenorrhea (46 cases for renal damage and 12 cases for high blood pressure). 
Table 3 Distribution of pathologies in the two groups

\begin{tabular}{llll}
\hline & Preterm infants n(\%) & Full-term n(\%) & P-Value \\
\hline Renal injury & $87(69.60)$ & $83(33.20)$ & $\mathrm{P}<0.00 \mathrm{I}$ \\
High blood pressure & $22(17.60)$ & $5(2.00)$ & $\mathrm{P}<0.001$ \\
Over weight & $40(32)$ & $20(8.0)$ & $\mathrm{P}=0.00002$ \\
\hline
\end{tabular}

\section{Correlation between the pathologies found}

We found a strong correlation between renal impairment and high blood pressure. Similarly, patients with renal impairment were 2.56 times more likely to develop high blood pressure.

\section{Logistic regression}

Neonatal infection, maternal secondary education, use of amino glycosides, aminophylline, cimetidine, ADEC vitamins and low APGAR score at the $5^{\text {th }}$ minute were associated with the onset of high blood pressure and renal injury. After the logistic regression, only neonatal infection, aminophylline use (since this was used only in preterm babies) and amino sides remained statistically significant and independently associated with these diseases (Table 4).

Table 4 Logistic regression

\begin{tabular}{|c|c|c|}
\hline Variables & Adjusted RR (95\% CI) & Adjusted p-value \\
\hline & \multicolumn{2}{|l|}{ Renal/HTA disease } \\
\hline $\begin{array}{l}\text { High school } \\
\text { education level }\end{array}$ & I,30 (0,7I-4,82) & $p=0,35$ \\
\hline Neonatal infection & $2,24(1,30-3,85)$ & $p=0,03$ \\
\hline Infectious risk & $0,40(0,18-0,88)$ & $p=0,45$ \\
\hline Aminophylline & $4,23(1,68-10,6)$ & $p=0,002$ \\
\hline Aminosides & $1,68(1,05-2,68)$ & $p=0,02$ \\
\hline $\begin{array}{l}\text { Anti } \mathrm{H} 2 \\
\text { (cimetidine) }\end{array}$ & I,80 (0,84-3,87) & $p=0,12$ \\
\hline Vitamin ADEC & I,87 (0,73-4,83) & $p=0,19$ \\
\hline NN asphyxia & $\mathrm{I}, 58(0,79-2,75)$ & $p=0,49$ \\
\hline $\begin{array}{l}\text { APGAR score } 5 \text { th } \\
\text { minute }\end{array}$ & $6,50(0,78-53,67)$ & $p=0,09$ \\
\hline
\end{tabular}

\section{Discussion}

\section{Limitation of the study}

The major limitation of this study was the use of urine deep stick to evaluate renal injury instead of carrying out assays for creatinine, cystatin $\mathrm{C}$ and microalbuminuria that could have helped us to appreciate the severity of the renal impairment.

\section{Socio-demographic and maternal data}

Of the 125 children born preterm and recruited in our series, both sexes were relatively equally represented with a sex ratio of 0.98 . The average age was 8.2 years with extremes ranging from 6 to 11 years. These findings are different from those of Kaze in 2017 and Grancher in France in $2014^{9,10}$ who found a clear predominance of the male sex. The mothers had an average age of 28.38 years, had mainly a secondary level of education and were mostly primiparous and single. High blood Pressure was the main maternal pathology associated with prematurity. These findings are similar to those of Grancher and collaborator in France who also found primiparity, an average age of 29.5 years and hypertension as the main maternal factors associated with prematurity. ${ }^{10}$ Bowers et al., ${ }^{11}$ on the other hand, found a higher level of education associated with preterm birth. ${ }^{11}$

\section{Hypertension in former premature infants cumulative incidence}

In our series, $57.60 \%$ of premature infants had blood pressure above the 90th percentile compared to $15.60 \%$ in children born at fullterm $(p<0,001)$. Amongst these, $40 \%$ had pre-hypertension and $17.6 \%$ had grade 1 hypertension $(p<0,001)$. We had a female predominance with a sex ratio of 0.75 . This prevalence was comparable, in different proportions, to the findings of the other authors, ${ }^{10,12-16}$ who also found high blood pressure in premature infants compared to fullterm controls. Bonamy et al., ${ }^{17}$ in 2017 in Switzerland, found that in children aged 6 years born before the 27th week of amenorrhoea, the blood pressure measured did not differ in the two groups. But when pr

ojectedonthereferencecurvesforage,sexandheight,therewasaslight increase in systolic blood pressure $(\mathrm{p}=0.02)$ and diastolic blood pressure $(\mathrm{p}=0.01)$ compared to controls. ${ }^{17}$ Namrakavashista in the USA in 2017 had a prevalence of $45.5 \%$ prehypertension compared to $19.29 \%$ in the control group $(\mathrm{p}=0.01)$ and a prevalence of hypertension grade $117.30 \%$ compared to $3.9 \%$ in the control group $(\mathrm{p}=0.001)$. Higher prevalence than ours, probably due to the high mortality of premature babies born before 30 weeks of amenorrhoea in our context. ${ }^{12}$ Grancher et al., ${ }^{10}$ in 2014 in France, found a prevalence of $51.9 \%$ among premature 2 -year-olds. ${ }^{10}$ On the other hand, our findings are much higher than those of Kaze et al., ${ }^{9}$ in 2018 in Cameroon, who found a prevalence of $9.51 \%$ among children born preterm/low birth weight, compared to $9.33 \%$ in the 2 to 5 years old ( $>>0.05$ ), with a discrete male predominance. ${ }^{9}$ This difference could be explained by different methodologies. Indeed, we were interested in children aged 6 to11, all prematurely born and recruited over a longer period (one year, compared to 6 months for Kaze'sstudy).

\section{The associated factors}

We did not find any factors associated with high blood pressure in our series. The prematurity alone could there fore increase the risk of increase in blood pressure in premature infants. This finding differ from the findings of Bonamy et al who found low gestational age, high body mass index and high heart rate, as factors associated with high blood pressure. ${ }^{17}$ While Namraka Vashishta found that low birth weight and young age at follow-up were associated with an increased risk of having a systolic or diastolic BP above the $90^{\text {th }}$ percentile. ${ }^{12}$ Satomi inomata et al., ${ }^{15}$ in 2015 in Japan, found that gestational age was positively associated with height and inversely associated with blood pressure at school age $(\mathrm{p}=0.04) .{ }^{15}$ Grancher et al in 2014 in France found primiparityandthebirthheadcircumferencebelowthe $10^{\text {th }}$ percentile significantly associated with abnormal blood pressure. ${ }^{10}$ Barker showed that low birth weight was associated with an increased risk of high blood pressure in adulthood; Therefore, prematurity could also be a risk factor for hypertension in adulthood. In fact, nephrogenesis 
is complete around the $36^{\text {th }}$ week of amenorrhea and the number of nephrons is reduced in premature infants, which can lead to hypertension at school age..$^{15}$

\section{Proteinuria as a marker of kidney disease in early preterm infants cumulative incidence}

Proteinuria was positive in 87 prematurely born patients, resulting in aprevalenceof $69.60 \%$ compared to $33.20 \%$ in the control group $(\mathrm{p}<0,001)$.Our findings are similar in different proportions to those of Kaze et al,. ${ }^{9}$ who found a prevalence of $25.4 \%$ of positive proteinuria in premature/small birth weight compared to $7.5 \%$ in controls $(\mathrm{p}=0.01){ }^{9}$

\section{The associated factors}

Positive proteinuria was strongly associated with neonatal infection, the use of amino sides and aminophylline in our series. The associated factors found in the literature vary from study to study. Our findings are different from those of the other authors. Kaze et al., ${ }^{9}$ found birth weight as a factor associated with renal impairment. ${ }^{9}$ On the other hand, Bachetta in France in 2009 found intrauterine and extra uterine growth retardation, and bronchopulmonary dysplasia in former premature infants was associated with a significant decrease in glomerular filtration rate and renal impact in the medium term. ${ }^{18}$

\section{Conclusion}

Renal impairment and high blood pressure were more frequently found in children born preterm. The presence of these conditions could compromise the subsequent cardiovascular prognosis. Strict compliance with post-admission follow-up of premature infants, as well as systematic research by health care provider, may have a better impact. It is therefore important that these pathologies be investigated during the daily monitoring of premature infants in order to detect them early and reduce avoidable morbidity in adulthood.

\section{Acknowledgments}

None.

\section{Conflicts of interest}

The authors do not declare any conflict of interest in relation to this article.

\section{Funding}

None.

\section{References}

1. Buitendijk S, Zeitlin J, Cuttini M, et al. Indicators of fetal and infant health outcomes. European Journal Of Obstetrics, Gynecology, and Reproductive Biology. 2003;111(1):66-77.

2. Slattery MM, Morrison JJ. Preterm delivery. Lancet. 2002;360(5):14891497.
3. Balaka B, Baeta S, Agbèrè Ad, et al. Facteurs De Risque Associés A La Prématurité Au Chu De Lomé, Togo. Bull Soc Pathol Exot. 2002;95(4):280-283.

4. Ye D, Kam Kl, Sanou I, et al. Epidemiological and evolutionary study of prematurity in the neonatology unit of the national hospital center -yo of ouagadougou (burkina faso). Annales De Pédiatrie. 1999;46(9):643649.

5. March of dimes, Pmnch, Save the Children. Who. Born Too Soon: The global action report on preterm birth. Geneva: World Health Organization; 2012.

6. Chiabi A, Mah E, Mvondo N, et al. Risk factors for premature births: a cross-sectional analysis of hospital records in a cameroonian health facility. Afr J Reprod Health. 2013;17(4):77-83.

7. Kerkhof Gf, Breukhoven PE, Leunissen RWJ, et al. Does preterm birth influence cardiovascular risk in early adulthood? J Pediatr. 2012;161(3):390-396.

8. Jaykaran Charan, Tamoghna Biswas. How To Calculate Sample Size For Different Study Designs In Medical Research? Indian journal of psychological medicine. 2013;35(2):121-126.

9. Kaze Folefack F, Itoua Ambando MS, Chelo D, et al. Effets de la prématurité et du petit poids de naissance sur la pression artérielle, la protéinurie et le débit de filtration glomérulaire chez les enfants de 2 a 5 ans. Néphrologie \& Thérapeutique. 2018;1(14):335 - 402.

10. Grancher Noémie. Evaluation de la tension artérielle a l'âge de deux ans des prématurés de moins de 33 semaines d'aménorrhée au chu de rouen. Faculté Mixte De Médecine Et De Pharmacie De Rouen; 2014.

11. Bowers K, Liu G, Wang P, et al. Birth weight, postnatal weight change, and risk for high blood pressure among chinese children. Pediatrics. 2011;127(5):E1272-E1279.

12. Vashishta N, Surapaneni V, Chawla S, et al. Association among prematurity $(<30$ weeks' gestational age), blood pressure, urinary albumin, calcium, and phosphate in early childhood. Pediatr Nephrol. 2017;32(7):1243-1250.

13. De Jong F, Monuteaux Mc, Van Elburg Rm, et al. Systematic review and meta-analysis of preterm birth and later systolic blood pressure. Hypertension. 2012;59(2):226-234.

14. Michael R Skilton, Jorma SA, Markus Juonala, et al. Fetal growth and preterm birth influence cardiovascular risk factors and arterial health in young adults the cardiovascular risk in young finns study. Arterioscler Thromb Vasc Biol. 2011;8(31):2975-2981.

15. Satomi Inomata, Taketoshi Yoshida, Uta Koura, et al. Effect of preterm birth on growth and cardiovascular disease risk at school age. Jpn Pediatr Soc. 2015;57:1126-1130.

16. Casey Crump, Jan Sundquist, Marilyn A Winkleby, et al. Preterm birth and risk of chronic kidney disease from childhood into mid-adulthood: national cohort study. Bmj. 2019;(365):1346.

17. Edstedt Bonamy A, Mohlkert L, Hallberg J, et al. Blood pressure in 6-year-old children born extremely preterm. $J$ Am Heart Assoc. 2017;6(8):E005858.

18. Bacchetta J, Cochat P. Le rein des anciens prématurés est-il menacé ? Arch Pédiatrie. 2008;15(7):1212-1222. 